\title{
THE SUPERCONDUCTING QUANTUM POINT CONTACT ${ }^{1}$
}

\author{
C. W. J. Beenakker ${ }^{a, b}$ and H. van Houten ${ }^{a}$ \\ ${ }^{a}$ Philips Research Laboratories \\ 5600 JA Eindhoven, The Netherlands \\ ${ }^{b}$ Institute for Theoretical Physics, University of California \\ Santa Barbara, CA 93106, USA
}

The theoretical prediction for the discretization of the supercurrent in a quantum point contact is reviewed in detail. A general relation is derived between the supercurrent and the quasiparticle excitation spectrum of a Josephson junction. The discrete spectrum of a quantum point contact between two superconducting reservoirs with phase difference $\delta \phi \in(-\pi / 2, \pi / 2)$ is shown to consist of a multiply degenerate state at energy $\Delta_{0} \cos (\delta \phi / 2)$ (one state for each of the $N$ propagating modes at the Fermi energy). The resulting zero-temperature supercurrent is $I=N\left(e \Delta_{0} / \hbar\right) \sin (\delta \phi / 2)$. The critical current is discretized in units of $e \Delta_{0} / \hbar$, dependent on the energy gap $\Delta_{0}$ of the bulk superconductor but not on the junction parameters. To achieve this analogue of the conductance quantization in the normal state, it is essential that the constriction is short compared to the superconducting coherence length.

\section{INTRODUCTION}

The work reviewed in this contribution was motivated by a sequence of analogies. First came the quantum point contact (QPC) for electrons $[1,2]$, a constriction in a two-dimensional electron gas with a quantized conductance. Then followed the optical analogue: The predicted [3] discretization of the transmission cross-section of a diffusely illuminated aperture or slit was recently observed experimentally [4]. One can speak of this optical analogue as a QPC for photons. Can one extend the analogue towards a QPC

${ }^{1}$ This research was supported in part by the National Science Foundation under Grant No. PHY89-04035. 
for Cooper pairs? The answer is Yes [5]. A narrow and short, impurityfree constriction in a superconductor has a zero-temperature critical current which is an integer multiple of $e \Delta_{0} / \hbar$, with $\Delta_{0}$ the energy gap of the bulk superconductor. In this paper we present our theory for the discretization of the supercurrent, in more detail than we could in our original publication [5]. For a less detailed, but more tutorial presentation, see Ref. [6]. To introduce the reader to the problem, we first summarize some basic facts on the Josephson effect in classical point contacts [7].

The theory of the stationary Josephson effect for a classical ballistic point contact is due to Kulik and Omel'yanchuk [8]. The adjectives classical and ballzstıc refer to the regime $\lambda_{\mathrm{F}} \ll W \ll l$, where $\lambda_{\mathrm{F}}$ is the Fermi wavelength, $W$ the width of the point contact, and $l$ the mean free path. The width $W$ and length $L$ of the constriction are also assumed to be much smaller than the superconducting coherence length $\xi_{0}=\hbar v_{\mathrm{F}} / \pi \Delta_{0}$ (with $v_{\mathrm{F}}$ the Fermi velocity). It is then irrelevant for the Josephson effect whether the constriction is made out of a superconductor or a normal metal. Kulik and Omel'yanchuk calculated the relationship between the supercurrent $I$ and the phase difference $\delta \phi$ of the pair potential in the superconducting reservoirs at opposite sides of the constriction. Their zero-temperature result is

$$
I=\pi G \frac{\Delta_{0}}{e} \sin (\delta \phi / 2),|\delta \phi|<\pi,
$$

where $G$ is the normal-state conductance of the point contact. For a three-dimensional (3D) point contact of cross-sectional area $S$ one has $G=\left(2 e^{2} / h\right) k_{\mathrm{F}}^{2} S / 4 \pi$, with $k_{\mathrm{F}}$ the Fermi wavevector. (A 2D point contact of width $W$ has $G=\left(2 e^{2} / h\right) k_{\mathrm{F}} W / \pi$.) These conductances, which are independent of the mean free path for $W \ll l$, are contact conductances. The current-phase relationship (1) is plotted in Fig. 1 (solid curve). As always, the Josephson current is an odd function of $\delta \phi$, and periodic with period $2 \pi$. The critical current $I_{\mathrm{c}} \equiv \max I(\delta \phi)$ is reached at $\delta \phi=\pi$, and equals $I_{\mathrm{c}}=\pi G \Delta_{0} / e$. At the critical current, the $I(\delta \phi)$ curve is discontinuous for $T=0$. This discontinuity is smoothed out at finite temperatures.

The supercurrent in the case of a ballistic point contact has a markedly different dependence on the phase than in the case of a tunnel junction, for which the Josephson effect was first proposed [10]. The $T=0$ current-phase relationship for a tunnel junction is given by $[11,12]$

$$
I=\frac{1}{2} \pi G \frac{\Delta_{0}}{e} \sin (\delta \phi),
$$

plotted as a dashed curve in Fig. 1. There is now no discontinuity in $I(\delta \phi)$. The critical current $I_{c}=\pi G \Delta_{0} / 2 e$ is reached at $\delta \phi=\pi / 2$. In both cases, $I_{\mathrm{c}}$ is proportional to $G$, but with different proportionality coefficients. The ratio $I_{\mathrm{c}} / G$ is therefore not universal. 


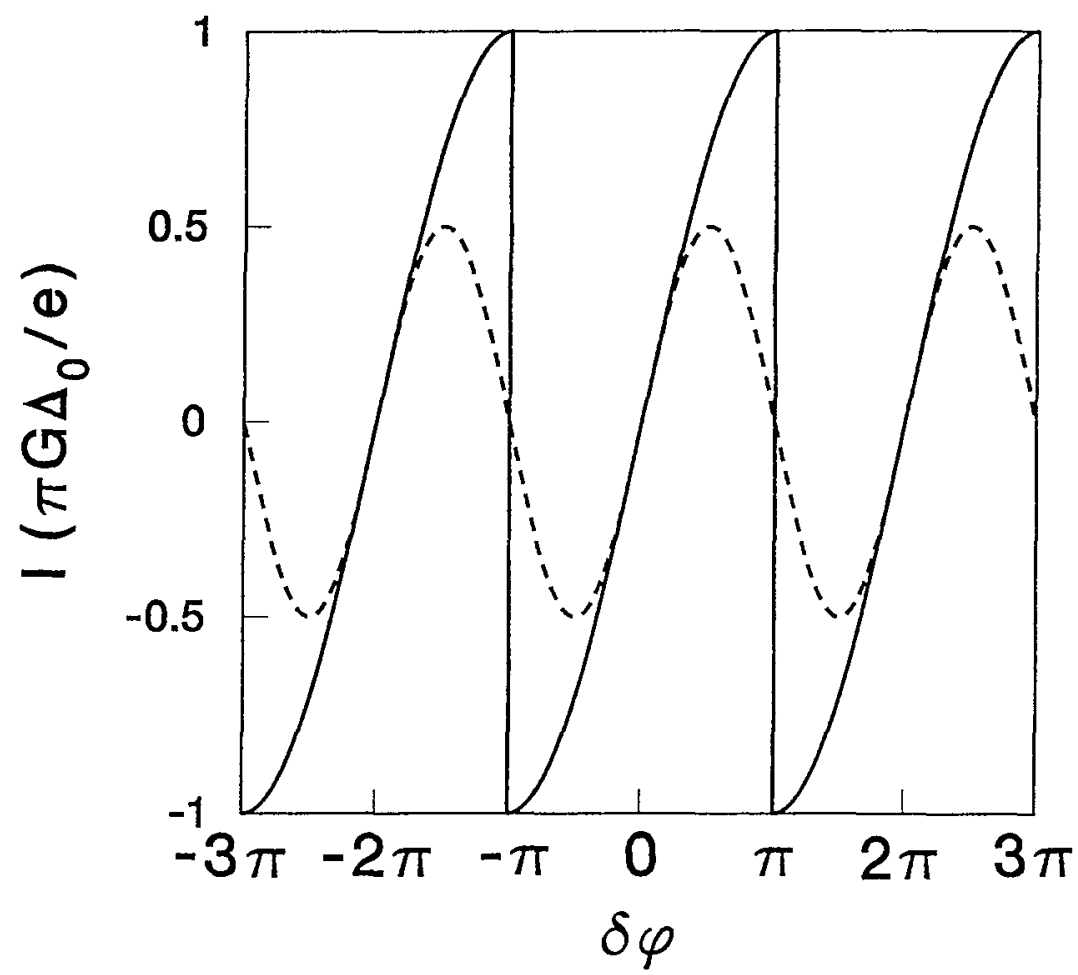

Figure 1: Current-phase relationship at $T=0$ of a classical ballistic point contact, according to Eq. (1) (solid curve), and of a tunnel junction, according to Eq. (2) (dashed curve).

Point contacts and tunnel junctions have in common that the length of the junction or weak link is much smaller than $\xi_{0}$. At low temperatures, the Josephson effect can occur also in junctions much longer than $\xi_{0}[13,14]$. Consider an SNS junction ( $\mathrm{S}=$ superconductor, $\mathrm{N}=$ normal metal) having a length $L_{\mathrm{N}}$ of the normal-metal region (i.e. the separation of the two SN interfaces) which greatly exceeds the coherence length $\xi_{0}$ of the superconductors (while still $L_{N} \ll l$ ). The current-phase relationship for the SNS junction at $T=0$ is a sawtooth $[15,16,17]$

$$
I=\alpha G \frac{\hbar v_{\mathrm{F}}}{e L_{\mathrm{N}}} \frac{\delta \phi}{\pi},|\delta \phi|<\pi,
$$

with critical current $I_{\mathrm{c}}=\alpha G \hbar v_{\mathrm{F}} / e L_{\mathrm{N}}$ (reached at $\delta \phi=\pi$ ). The numerical coefficient $\alpha$ is of order unity and depends on the dimensionality of the junction. (There is a disagreement among Refs. [15] and [17] on the precise 
value of $\alpha$.) The $I_{\mathrm{c}} / G$ ratio is now a function of $L_{\mathrm{N}}$. This is in contrast to the point contact and tunnel junction, where $I_{\mathrm{c}} / G$ depends only on the gap $\Delta_{0}$ in the bulk reservoir-not on the geometry of the junction. There is also a different temperature dependence. The supercurrents (1) and (2) decay when $T$ approaches the critical temperature $T_{\mathrm{c}} \simeq \Delta_{0} / k_{\mathrm{B}}$, wheras Eq. (3) requires temperatures smaller than $T_{\mathrm{c}}$ by a factor $\xi_{0} / L_{\mathrm{N}} \ll 1$.

The problem addressed in Ref. [5] was to find the superconducting analogue of the conductance quantization in the normal state, which is a geometry-independent effect. The system in which we looked for such an effect was a short ballistic point contact, much shorter than $\xi_{0}$, and of a width comparable to $\lambda_{\mathrm{F}}$. One would hope that a quantized $G$ will lead to a "quantization" (or, more correctly, a discretization) of $I_{\mathrm{c}}$ in units which will still depend on $\Delta_{0}$, but which are independent of the properties of the junction. The long SNS junction is not a likely candidate because of its $L_{\mathrm{N}}$-dependent critical current. Still, if a quantum point contact is inserted in a long SNS junction $\left(L_{\mathrm{N}} \gg \xi_{0}\right)$, one might expect to find geometry-dependent quantum size effects on the Josephson current. Theoretical work on that structure was done by Furusaki, Takayanagi, and Tsukada [18]. Their result is that $I_{c}$ increases stepwise with increasing width for certain shapes of the constriction, but not generically. When steps do occur, the step height depends sensitively on the parameters of the junction. As we will show, a short junction (short compared to $\xi_{0}$ ) is essential for a generic, junction-independent behavior.

In Sec. II we describe the method that we use to calculate the Josephson current through a quantum point contact, which is different from the method used in Ref. [8] for a classical point contact. The central result of that section is a relation between the supercurrent and the quasiparticle excitation spectrum of the Josephson junction. In Sec. III the actual calculation is presented, following Ref. [5]. We conclude in Sec. IV with a brief discussion of a possible experimental realization of the superconducting quantum point contact.

The present theory is based on two approximations: Firstly, we assume adiabatic transport, i.e. absence of scattering between the transverse modes (or 1D subbands) in the quantum point contact. The assumption of adiabaticity brings out the essential features of the effect in the simplest and most direct way, just as it does in the normal state [19]. As in the normal state [20], we expect the discretization of the Josephson current to be robust to deviations from adiabaticity which do not cause backscattering. In particular, although we assume both $l \gg W$ and $l \gg \xi_{0}$ in the present analysis, we believe that the condition $l \gg W$ on the mean free path is sufficient, since scattering in the wide regions outside the constriction is unlikely to cause backscattering into the constriction.

Secondly, in order to further simplify the problem, we treat the propa- 
gation of the modes in the WKB approximation. The WKB approximation breaks down if the Fermi energy $E_{\mathrm{F}}$ lies within $\Delta_{0}$ from the cutoff energy of a transverse mode (being the $1 \mathrm{D}$ subband bottom). We can therefore only describe the plateau region of the discretized Josephson current, not the transition from one multiple of $e \Delta_{0} / \hbar$ to the next. Since the transition region is smaller than the plateau region by a factor of order $N \Delta_{0} / E_{\mathrm{F}}$ (where $N$ is the number of occupied subbands in the constriction), it is relatively unimportant if $\Delta_{0} \ll E_{\mathrm{F}}$ and $N \simeq 1$.

\section{JOSEPHSON CURRENT FROM EXCITATION SPECTRUM}

The theory of Kulik and Omel'yanchuk [8] on the Josephson effect in a classical point contact is based on a classical Boltzmann-type transport equation for the Green's functions (the Eilenberger equation [7]), which is not applicable to a quantum point contact. The present analysis is based on the fully quantum-mechanical Bogoliubov-de Gennes (BdG) equations for quasiparticle wavefunctions, into which the Green's functions can be expanded [21]. In the present section we describe how the Josephson current can be obtained directly from the quasi-particle excitation spectrum-without having to calculate the Green's functions. This method is particularly well suited for the point contact Josephson junction, which has an excitation spectrum of a very simple form.

The $\mathrm{BdG}$ equations consist of two Schrödinger equations for electron and hole wavefunctions $\mathrm{u}(\mathbf{r})$ and $\mathrm{v}(\mathbf{r})$, coupled by the pair potential $\Delta(\mathbf{r})$ :

$$
\left(\begin{array}{cc}
\mathcal{H} & \Delta \\
\Delta^{*} & -\mathcal{H}^{*}
\end{array}\right)\left(\begin{array}{c}
\mathrm{u} \\
\mathrm{v}
\end{array}\right)=\epsilon\left(\begin{array}{c}
\mathrm{u} \\
\mathrm{v}
\end{array}\right)
$$

where $\mathcal{H}=(\mathbf{p}+e \mathbf{A})^{2} / 2 m+V-E_{\mathrm{F}}$ is the single-electron Hamiltonian in the field of a vector potential $\mathbf{A}(\mathbf{r})$ and electrostatic potential $V(\mathbf{r})$. The excitation energy $\epsilon$ is measured relative to the Fermi energy. Since the matrix operator in Eq. (4) is hermitian, the eigenfunctions $\Psi=(\mathrm{u}, \mathrm{v})$ form a complete orthonormal set. One readily verifies that if $(u, v)$ is an eigenfunction with eigenvalue $\epsilon$, then $\left(-v^{*}, u^{*}\right)$ is also an eigenfunction, with eigenvalue $-\epsilon$. The complete set of eigenvalues thus lies symmetrically around zero. The excitation spectrum consists of all positive $\epsilon$.

In a uniform system with $\Delta(\mathbf{r}) \equiv \Delta_{0} \mathrm{e}^{\mathrm{i} \phi}, \mathbf{A}(\mathbf{r}) \equiv 0, V(\mathbf{r}) \equiv 0$, the solution of the $\mathrm{BdG}$ equations is

$$
\begin{aligned}
\epsilon & =\left(\left(\hbar^{2} k^{2} / 2 m-E_{\mathrm{F}}\right)^{2}+\Delta_{0}^{2}\right)^{1 / 2} \\
\mathbf{u}(\mathbf{r}) & =(2 \epsilon)^{-1 / 2} \mathrm{e}^{\mathrm{i} \phi / 2}\left(\epsilon+\hbar^{2} k^{2} / 2 m-E_{\mathbf{F}}\right)^{1 / 2} \mathrm{e}^{\mathrm{i} \mathbf{k} \cdot \mathbf{r}}, \\
\mathbf{v}(\mathbf{r}) & =(2 \epsilon)^{-1 / 2} \mathrm{e}^{-\mathrm{i} \phi / 2}\left(\epsilon-\hbar^{2} k^{2} / 2 m+E_{\mathrm{F}}\right)^{1 / 2} \mathrm{e}^{\mathrm{i} \mathbf{k} \cdot \mathbf{r}} .
\end{aligned}
$$


The excitation spectrum is continuous, with excitation gap $\Delta_{0}$. The eigenfunctions $(\mathrm{u}, \mathrm{v})$ are plane waves characterized by a wavevector $\mathrm{k}$. The coefficients of the plane waves are the two coherence factors of the BCS theory. As we will discuss in the next section, the excitation spectrum acquires a discrete part in the presence of a Josephson junction. The discrete spectrum corresponds to bound states in the gap $\left(\epsilon<\Delta_{0}\right)$, localized within $\xi_{0}$ from the junction. The Josephson current turns out to be essentially determined by the discrete part of the excitation spectrum.

Close to the junction the pair potential is not uniform. To determine $\Delta(\mathbf{r})$ one has to solve the self-consistency equation [21]

$$
\Delta(\mathbf{r})=g(\mathbf{r}) \sum_{\epsilon>0} \mathrm{v}^{*}(\mathbf{r}) \mathrm{u}(\mathbf{r})[1-2 f(\epsilon)],
$$

where the sum is over all states with positive eigenvalue, ${ }^{2}$ and $f(\epsilon)=$ $\left[1+\exp \left(\epsilon / k_{\mathrm{B}} T\right)\right]^{-1}$ is the Fermi function. The coefficient $g$ is minus the interaction constant of the BCS theory of superconductivity. At an SN interface, $g$ changes abruptly (over atomic distances) from a positive constant to zero. In contrast, the Cooper pair density $\Delta / g$ goes to zero only over macroscopic distances (on the order of $\xi_{0}$ for a planar SN interface). Because of Eq. (8), the determination of the excitation energy spectrum is a non-linear problem, which is further complicated by the fact that the vector potential has also to be determined self-consistently from the current density, via Maxwell's equations. (The electrostatic potential can usually be assumed to be the same as in the normal state.)

Once the eigenvalue problem (4) is solved self-consistently, one can calculate the equilibrium average of a single-electron operator $\mathcal{P}$ by means of the formula (cf. Ref. [21])

$$
\langle\mathcal{P}\rangle=2 \sum_{\epsilon>0} \int d \mathbf{r}\left(f(\epsilon) \mathbf{u}^{*} \mathcal{P} \mathrm{u}+[1-f(\epsilon)] \mathbf{v} \mathcal{P} \mathbf{v}^{*}\right)
$$

Notice the reverse order, $\mathrm{u}^{*} \mathcal{P} \mathrm{u}$ versus ${ }_{\mathrm{v}} \mathcal{P}_{\mathrm{v}^{*}}$, and the different thermal weight factors, $f(\epsilon)$ for electrons and $f(-\epsilon)=1-f(\epsilon)$ for holes. The prefactor of 2 accounts for both spin directions. (It is assumed that $\mathcal{P}$ does not couple to the spin.) We are interested in particular in the equilibrium current density $\mathbf{j}(\mathbf{r})$, which is given by

$$
\mathbf{j}=-2 \frac{e}{m} \sum_{\epsilon>0} \operatorname{Re}\left(f(\epsilon) \mathrm{u}^{*}(\mathbf{p}+e \mathbf{A}) \mathrm{u}+[1-f(\epsilon)] \mathrm{v}(\mathbf{p}+e \mathbf{A}) \mathrm{v}^{*}\right),
$$

in accordance with Eq. (9). The Josephson current $I$ is the integral of $\mathbf{j} \cdot \mathbf{n}$ over the cross-section of the junction (with $\mathbf{n}$ a unit vector perpendicular to the cross-section).

\footnotetext{
${ }^{2} \mathrm{~A}$ cutoff at $\hbar \omega_{\mathrm{D}}$, with $\omega_{\mathrm{D}}$ the Debije frequency, has to be introduced as usual in the BCS theory.
} 
There is an alternative (and often more convenient) way to arrive at the total equilibrium current $I$ flowing through the Josephson junction, which is to use the fundamental relation [11]

$$
I=\frac{2 e}{\hbar} \frac{d F}{d \delta \phi}
$$

between the Josephson current and the derivative of the free energy $F$ with respect to the phase difference. (The derivative is to be taken without varying the vector potential.) To apply this relation we need to know how to obtain $F$ from the BdG equations. This is somewhat tricky, since (because of the pair potential) one can not express $F$ exclusively in terms of the excitation energies (as one can for non-interacting particles). The required formula was derived by Bardeen et al. [23] from the Green's function expression for $F$. Here we present an alternative derivation, directly from the BdG equations.

Following De Gennes [21], we write

$$
\begin{aligned}
& F=U-T S \\
& U=\langle\mathcal{H}\rangle+U_{\mathrm{int}}, \\
& U_{\mathrm{int}}=-\int d \mathbf{r}|\Delta|^{2} / g, \\
& S=-2 k_{\mathrm{B}} \sum_{\epsilon>0}[f \ln f+(1-f) \ln (1-f)] .
\end{aligned}
$$

The energy $U$ is the sum of the single-particle energy $\langle\mathcal{H}\rangle$ [defined according to Eq. (9)] and the interaction-potential energy $U_{\text {int }}$ (which is negative, since the interaction is attractive). The entropy $S$ of the independent fermionic excitations can be rewritten as

$$
S=-2 k_{\mathrm{B}} \sum_{\epsilon>0}\left[\ln f+(1-f) \epsilon / k_{\mathrm{B}} T\right] .
$$

Using Eqs. (4) and (8) we obtain for the single-particle energy the expression

$$
\langle\mathcal{H}\rangle=\sum_{\epsilon>0}[\epsilon f-\epsilon(1-f)]-2 U_{\mathrm{mt}}+\sum_{\epsilon>0} \epsilon \int d \mathbf{r}\left(|\mathrm{u}|^{2}-|\mathrm{v}|^{2}\right)
$$

The term containing the u's and v's is in fact independent of these eigenfunctions, as one sees from the following sequence of equalities:

$$
\begin{aligned}
\sum_{\epsilon>0} \epsilon \int d \mathbf{r}\left(|\mathrm{u}|^{2}-|\mathrm{v}|^{2}\right) & =\sum_{\epsilon>0} \int d \mathbf{r}\left(\mathrm{u}^{*} \mathcal{H} \mathrm{u}+\mathrm{v} \mathcal{H} \mathrm{v}^{*}\right) \\
& =\frac{1}{2} \sum_{\epsilon} \int d \mathbf{r}\left(\mathrm{u}^{*} \mathcal{H} \mathrm{u}+\mathrm{v}^{*} \mathcal{H}^{*} \mathrm{v}\right) \\
& =\frac{1}{2} \operatorname{Tr}\left(\begin{array}{cc}
\mathcal{H} & 0 \\
0 & \mathcal{H}^{*}
\end{array}\right)=\operatorname{Tr} \mathcal{H}
\end{aligned}
$$


Here we have made use of the completeness of the set of eigenfunctions $(u, v)$ when both positive and negative $\epsilon$ are included. Collecting results, the expression for the free energy becomes

$$
F=-2 k_{\mathrm{B}} T \sum_{\epsilon>0} \ln \left[2 \cosh \left(\epsilon / 2 k_{\mathrm{B}} T\right)\right]+\int d \mathbf{r}|\Delta|^{2} / g+\operatorname{Tr} \mathcal{H}
$$

This is the electronic contribution to the free energy, without the energy stored in the magnetic field. Eq. (19) agrees with Ref. [23] - except for the term $\operatorname{Tr} \mathcal{H}$, which is absent in their expression for the free energy. ${ }^{3}$

The first term in Eq. (19) (the sum over $\epsilon$ ) can be formally interpreted as the free energy of non-interacting electrons, all of one single spin, in a "semiconductor" with Fermi level halfway between the "conduction band" (positive $\epsilon$ ) and the "valence band" (negative $\epsilon$ ). This familiar [22] semiconductor model of a superconductor appeals to intuition, but does not give the free energy correctly. The second term $\left(-U_{\text {int }}\right)$ in Eq. (19) corrects for a double-counting of the interaction energy in the semiconductor model. The third term $(\operatorname{Tr} \mathcal{H})$ cancels a divergence at large $\epsilon$ of the series in the first term. Substituting $F$ into Eq. (11), we obtain the required expression for the Josephson current:

$$
\begin{aligned}
I= & -\frac{2 e}{\hbar} \sum_{p} \tanh \left(\epsilon_{p} / 2 k_{\mathrm{B}} T\right) \frac{d \epsilon_{p}}{d \delta \phi} \\
& -\frac{2 e}{\hbar} 2 k_{\mathrm{B}} T \int_{\Delta_{0}}^{\infty} d \epsilon \ln \left[2 \cosh \left(\epsilon / 2 k_{\mathrm{B}} T\right)\right] \frac{d \rho}{d \delta \phi} \\
& +\frac{2 e}{\hbar} \frac{d}{d \delta \phi} \int d \mathbf{r}|\Delta|^{2} / g,
\end{aligned}
$$

where we have rewritten $\sum_{\epsilon>0}$ as a sum over the discrete positive eigenvalues $\epsilon_{p}(p=1,2, \ldots)$, and an integration over the continuous spectrum with density of states $\rho(\epsilon)$. The term $\operatorname{Tr} \mathcal{H}$ in $\mathrm{Eq}$. (19) does not depend on $\delta \phi$, and therefore does not contribute to $I$. The term $-U_{\text {int }}$ does contribute in general. For the case of a point contact Josephson junction considered in this paper, however, we will show that this contribution (the third term in Eq. (20) can be neglected relative to the contribution from the semiconductor model. A calculation of the Josephson current from Eq. (20) then requires only knowledge of the eigenvalues. This is in contrast to a calculation based on Eq. (10), which requires also the eigenfunctions.

\footnotetext{
${ }^{3}$ Bardeen et al. [23] use the free energy (including the magnetic field contribution) to determine the self-consistent pair and vector potentials variationally: The self-consistent $\Delta$ and $\mathbf{A}$ minimize the total free encrgy of the system. Since $\operatorname{Tr} \mathcal{H}$ depends on $\mathbf{A}$, it is not immediately obvious to us that it is justified to disregard this term in the variational calculation of $\dot{A}$, as was done in Ref. [23]. A possible argument is that energies near the Fermi energy do not contribute to $\operatorname{Tr} \mathcal{H}$, because eigenvalues below $E_{\mathrm{F}}$ cancel those above $E_{\mathrm{F}}$. The cancellation is not exact, however.
} 


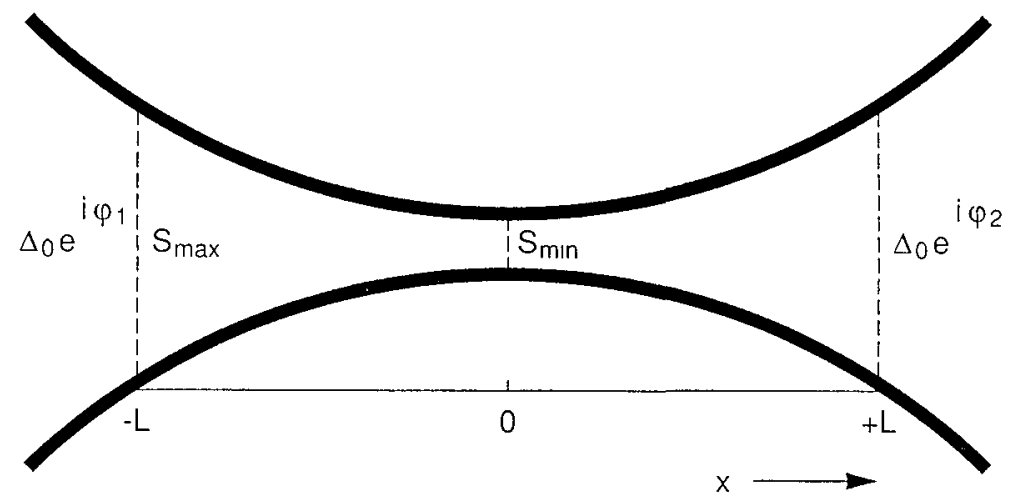

Figure 2: Schematic drawing of a superconducting constriction of slowly varying width. (Taken from Ref. [5].)

\section{JOSEPHSON CURRENT THROUGH A QUANTUM POINT CONTACT}

Let us now consider an impurity-free superconducting constriction (Fig. 2), whose cross-sectional area $S(x)$ widens from $S_{\min }$ to $S_{\max } \gg S_{\min }$ over a length $L \gg \lambda_{F}$ and $\ll \xi_{0}$. The $x$-axis is along the constriction, and the area $S_{\max }$ is reached at $x= \pm L$. We are interested in the case that only a small number $N \simeq S_{\mathrm{min}} / \lambda_{\mathrm{F}}^{2}$ of transverse modes can propagate through the constriction at energy $E_{\mathbf{F}}$, and we assume that the propagation is adıabatic, i.e. without scattering between the modes. In the superconducting reservoirs to the left and right of the constriction the pair potential (of absolute value $\Delta_{0}$ ) has phase $\phi_{1}$ and $\phi_{2}$, respectively. We wish to calculate the current $I(\delta \phi)$ which flows in equilibrium through the constriction, for a given (timeindependent) phase difference $\delta \phi \equiv \phi_{1}-\phi_{2} \in(-\pi, \pi)$ between the two reservoirs. The characterization of the reservoirs by a constant phase is not strictly correct. The phase of the pair potential has a gradient in the bulk if a current flows. The gradient is $1 / \xi_{0}$ when the current density equals the critical current density in the bulk. In our case the critical current is limited by the point contact, so that the gradient of the phase in the bulk is much smaller than $1 / \xi_{0}$ (by a factor $S_{\operatorname{mm}} / S(x) \ll 1$ ). Since the excitation spectrum is determined by the region within $\xi_{0}$ from the junction, one can safely neglect this gradient in calculating $I(\delta \phi)$ from Eq. (20).

As we enter the constriction from, say, the left reservoir, the pair potential $\Delta(\mathbf{r})$ begins to vary from the bulk value $\Delta_{0} \exp \left(i \phi_{1}\right)$, for two reasons: (a) because the small number of transverse modes leads to a non-uniform density; and (b) because of contributions from the $N$ modes which have reached $\mathbf{r}$ from the right reservoir. Non-uniformities in $\Delta$ due to $(a)$ and 
(b) are of order $1 / N(x) \simeq \lambda_{\mathrm{F}}^{2} / S(x)$ and $N / N(x) \simeq S_{\mathrm{min}} / S(x)$, respectively $(N(x)$ being the number of propagating modes at $x$ ). For $|x|>L$ we have both $S(x) \gg \lambda_{F}^{2}$ and $S(x) \gg S_{m n}$. One may therefore neglect these nonuniformities for $|x|>L$, and put

$$
\Delta(\mathbf{r})= \begin{cases}\Delta_{0} \mathrm{e}^{1 \phi_{1}} & \text { if } x<-L \\ \Delta_{0} \mathrm{e}^{1 \phi_{2}} & \text { if } x>L\end{cases}
$$

Note the difference with planar SNS junctions, where $\Delta$ recovers its bulk value only at a distance $\xi_{0}$ from the SN interface. The much shorter decay length for non-uniformities due to a constriction is a geometrical "dilution" effect, well known in the theory of weak links $[7,8]$. No specific assumptions are made on the variation of $\Delta$ in the narrow part of the constriction. In particular, our analysis also applies to a non-superconducting constriction $\left(\Delta(\mathbf{r})=0\right.$ at $\left.S_{\min }\right)$. Eq. (21) remains valid up to terms of order $S_{\min } / S_{\max } \ll$ 1 .

We describe the propagation of quasiparticles through the constriction by means of the BdG equations (4). The electrostatic potential $V(\mathbf{r})$ is now the confining potential which defines the constriction. We neglect the vector potential induced by the Josephson current. This is allowed if the total flux penetrating the junction is small compared to the flux quantum $h / 2 e$, which is generally the case if the constriction is small compared to $\xi_{0} \cdot{ }^{4}$ For $\mathbf{A}(\mathbf{r}) \equiv$ 0 , the transverse modes $|n\rangle \equiv \phi_{n}(\mathbf{r})$ are eigenfunctions of $\left(p_{y}^{2}+p_{z}^{2}\right) / 2 m+V(\mathbf{r})$, with eigenvalues $E_{n}(x)$. We expand $\Psi(\mathbf{r})=\sum_{n}\left(\mathrm{u}_{n}, \mathrm{v}_{n}\right) \phi_{n}$ into transverse modes and neglect off-diagonal matrix elements $\left\langle n|\mathcal{H}| n^{\prime}\right\rangle$ and $\left\langle n|\Delta| n^{\prime}\right\rangle(\langle\ldots\rangle$ denotes integration over $y$ and $z$ ). This is the adiabatic approximation. The functions $\mathrm{u}_{n}(x)$ and $\mathrm{v}_{n}(x)$ then satisfy the one-dimensional BdG equations

$$
\begin{aligned}
\left(p_{x}^{2} / 2 m-U_{n}\right) \mathrm{u}_{n}+\Delta_{n} \mathrm{v}_{n} & =\epsilon \mathrm{u}_{n}, \\
-\left(p_{x}^{2} / 2 m-U_{n}\right) \mathrm{v}_{n}+\Delta_{n}^{*} \mathrm{u}_{n} & =\epsilon \mathrm{v}_{n},
\end{aligned}
$$

where $U_{n}(x)=E_{\mathrm{F}}-E_{n}(x)-(1 / 2 m)\left\langle n\left|p_{x}^{2}\right| n\right\rangle \approx E_{\mathrm{F}}-E_{n}(x)$ is the kinetic energy of motion along the constriction in mode $n$, and $\Delta_{n}(x)=\langle n|\Delta| n\rangle$ is the projection of $\Delta(\mathbf{r})$ onto the $n$-th mode. We will consider one mode $n \leq N$ at a time, and omit the subscript $n$ for notational simplicity in most of the equations.

In order to simplify the solution of the 1D BdG equations (22), we adopt the WKB method of Bardeen et al. [23], which consists in substituting

$$
\left(\begin{array}{l}
\mathrm{u} \\
\mathrm{v}
\end{array}\right)=\left(\begin{array}{c}
\mathrm{e}^{i \eta / 2} \\
\mathrm{e}^{-i \eta / 2}
\end{array}\right) \exp \left(\mathrm{i} \int_{0}^{x} k\left(x^{\prime}\right) d x^{\prime}\right)
$$

${ }^{4}$ More precisely, the constriction should be small compared to the penetration depth of the magnetic field in the Josephson junction, which length is much larger than $\xi_{0}$ in a type II superconductor [7]. 
into Eq. (22) and neglecting second order derivatives (or products of first order derivatives). One thus generalizes the familiar WKB method for the Schrödinger equation to the BdG equations, by having not only a spatially dependent wavevector $k(x)$-but also spatially dependent coherence factors $\exp [ \pm i \eta(x) / 2]$. The resulting equations for $\eta(x)$ and $k(x)$ are

$$
\begin{aligned}
-\left(\hbar^{2} / 2 m\right) k \eta^{\prime}+\epsilon & =|\Delta| \cos (\eta-\phi), \\
\left(\hbar^{2} / 2 m\right)\left(k^{2}-i k^{\prime}\right)-U & =\mathrm{i}|\Delta| \sin (\eta-\phi),
\end{aligned}
$$

where $\Delta(x) \equiv|\Delta(x)| \mathrm{e}^{\mathrm{l} \phi(x)}$. In general, both $\eta$ and $k$ are complex. The WKB approximation requires that $U$ changes slowly on the scale of $\lambda_{F}$, so that reflections due to abrupt variations in the confining potential can be neglected. Reflections (accompanied by a change in sign of Re $k$ ) due to spatial variations in the pair potential are negligible provided that $|\Delta|$ is much smaller than the kinetic energy $U$ of motion along the constriction. Since $U \gtrsim E_{\mathrm{F}}-E_{N}(0)$, the WKB method can not treat the threshold regime that $E_{\mathrm{F}}$ lies within $\Delta_{0}$ from the cutoff energy $E_{N}(0)$ of the highest mode $N$ at the narrowest point of the constriction $(x=0)$. The energy separation $\delta E \equiv E_{N+1}(0)-E_{N}(0) \simeq E_{\mathrm{F}} / N$ is much larger than $\Delta_{0}$ for small $N$, so that the threshold regime $\left|E_{\mathrm{F}}-E_{N}(0)\right| \lesssim \Delta_{0}$ consists only of small intervals in Fermi energy (smaller than the non-threshold intervals by a factor $\Delta_{0} / \delta E \ll$ $1)$.

For $|x|>L$, where $\Delta$ is independent of $x$, one has a constant $\eta$ which can take on the two values $\eta^{\mathrm{e}}$ and $\eta^{\mathrm{h}}$,

$$
\eta^{\mathrm{e}, \mathrm{h}}=\phi+\sigma^{\mathrm{e}, \mathrm{h}} \arccos \left(\epsilon / \Delta_{0}\right),
$$

where $\sigma^{\mathrm{e}} \equiv 1, \sigma^{\mathrm{h}} \equiv-1$. We have $\phi=\phi_{1}$ for $x<-L$ and $\phi=\phi_{2}$ for $x>L$. The function arccost is defined such that $\arccos t \in(0, \pi / 2)$ for $0<t<1$; for $t>1$, one has $\mathrm{i} \arccos t=\ln \left[t+\left(t^{2}-1\right)^{1 / 2}\right]$. The (unnormalized) WKB wavefunctions $\Psi_{ \pm}^{\mathrm{e}, \mathrm{h}}(x)$ for $|x|>L$ describe an electron-like (e) or hole-like (h) quasiparticle with positive $(+)$ or negative $(-)$ wavevector,

$$
\begin{aligned}
\Psi_{ \pm}^{\mathrm{e}, \mathrm{h}} & =\left(k^{\mathrm{e}, \mathrm{h}}\right)^{-1 / 2}\left(\begin{array}{c}
\mathrm{e}^{\mathrm{i} \eta^{\mathrm{e}, \mathrm{h}} / 2} \\
\mathrm{e}^{-1 \eta^{\mathrm{e}, \mathrm{h}} / 2}
\end{array}\right) \exp \left( \pm \mathrm{i} \int_{0}^{x} k^{\mathrm{e}, \mathrm{h}} d x^{\prime}\right) \\
k^{\mathrm{e}, \mathrm{h}} & =\left(2 m / \hbar^{2}\right)^{1 / 2}\left[U+\sigma^{\mathrm{e}, \mathrm{h}}\left(\epsilon^{2}-\Delta_{0}^{2}\right)^{1 / 2}\right]^{1 / 2}
\end{aligned}
$$

The square roots are to be taken such that $\operatorname{Re} k^{\mathrm{e}, \mathrm{h}} \geq 0, \operatorname{Im} k^{\mathrm{e}} \geq 0, \operatorname{Im} k^{\mathrm{h}} \leq 0$. The wavefunction (27) is a solution for $|x|>L$ of the BdG equations up to second order derivatives. One can verify that for zero confining potential the solution (27), (28) is equivalent to the energy spectrum and coherence factors given in Eqs. (5)-(7).

For $\epsilon>\Delta_{0}$, the wavevectors $k^{e, h}$ are real at $|x|>L$, and hence the wavefunctions (27) remain properly bounded as $|x| \rightarrow \infty$. This is the continuous spectrum. The density of states $\rho^{\mathrm{e}, \mathrm{h}}(\epsilon)$ of the electron and hole 
into Eq. (22) and neglecting second order derivatives (or products of first order derivatives). One thus generalizes the familiar WKB method for the Schrödinger equation to the BdG equations, by having not only a spatially dependent wavevector $k(x)$-but also spatially dependent coherence factors $\exp [ \pm \mathrm{i} \eta(x) / 2]$. The resulting equations for $\eta(x)$ and $k(x)$ are

$$
\begin{aligned}
-\left(\hbar^{2} / 2 m\right) k \eta^{\prime}+\epsilon & =|\Delta| \cos (\eta-\phi), \\
\left(\hbar^{2} / 2 m\right)\left(k^{2}-i k^{\prime}\right)-U & =\mathrm{i}|\Delta| \sin (\eta-\phi),
\end{aligned}
$$

where $\Delta(x) \equiv|\Delta(x)| \mathrm{e}^{1 \phi(x)}$. In general, both $\eta$ and $k$ are complex. The WKB approximation requires that $U$ changes slowly on the scale of $\lambda_{F}$, so that reflections due to abrupt variations in the confining potential can be neglected. Reflections (accompanied by a change in sign of Re $k$ ) due to spatial variations in the pair potential are negligible provided that $|\Delta|$ is much smaller than the kinetic energy $U$ of motion along the constriction. Since $U \gtrsim E_{\mathrm{F}}-E_{N}(0)$, the WKB method can not treat the threshold regime that $E_{\mathrm{F}}$ lies within $\Delta_{0}$ from the cutoff energy $E_{N}(0)$ of the highest mode $N$ at the narrowest point of the constriction $(x=0)$. The energy separation $\delta E \equiv E_{N+1}(0)-E_{N}(0) \simeq E_{\mathrm{F}} / N$ is much larger than $\Delta_{0}$ for small $N$, so that the threshold regime $\left|E_{\mathrm{F}}-E_{N}(0)\right| \lesssim \Delta_{0}$ consists only of small intervals in Fermi energy (smaller than the non-threshold intervals by a factor $\Delta_{0} / \delta E \ll$ 1).

For $|x|>L$, where $\Delta$ is independent of $x$, one has a constant $\eta$ which can take on the two values $\eta^{\mathrm{e}}$ and $\eta^{\mathrm{h}}$,

$$
\eta^{\mathrm{e}, \mathrm{h}}=\phi+\sigma^{\mathrm{e}, \mathrm{h}} \arccos \left(\epsilon / \Delta_{0}\right)
$$

where $\sigma^{\mathrm{e}} \equiv 1, \sigma^{\mathrm{h}} \equiv-1$. We have $\phi=\phi_{1}$ for $x<-L$ and $\phi=\phi_{2}$ for $x>L$. The function $\arccos t$ is defined such that $\arccos t \in(0, \pi / 2)$ for $0<t<1$; for $t>1$, one has i arccos $t=\ln \left[t+\left(t^{2}-1\right)^{1 / 2}\right]$. The (unnormalized) WKB wavefunctions $\Psi_{ \pm}^{\mathrm{e}, \mathrm{h}}(x)$ for $|x|>L$ describe an electron-like (e) or hole-like (h) quasiparticle with positive $(+)$ or negative $(-)$ wavevector,

$$
\begin{aligned}
\Psi_{ \pm}^{\mathrm{e}, \mathrm{h}} & =\left(k^{\mathrm{e}, \mathrm{h}}\right)^{-1 / 2}\left(\begin{array}{c}
\mathrm{e}^{\mathrm{i} \eta^{\mathrm{e}, \mathrm{h}} / 2} \\
\mathrm{e}^{-\mathrm{i} \eta^{e, \mathrm{~h}} / 2}
\end{array}\right) \exp \left( \pm \mathrm{i} \int_{0}^{x} k^{\mathrm{e}, \mathrm{h}} d x^{\prime}\right) \\
k^{\mathrm{e}, \mathrm{h}} & =\left(2 m / \hbar^{2}\right)^{1 / 2}\left[U+\sigma^{\mathrm{e}, \mathrm{h}}\left(\epsilon^{2}-\Delta_{0}^{2}\right)^{1 / 2}\right]^{1 / 2}
\end{aligned}
$$

The square roots are to be taken such that $\operatorname{Re} k^{\mathrm{e}, \mathrm{h}} \geq 0, \operatorname{Im} k^{\mathrm{e}} \geq 0, \operatorname{Im} k^{\mathrm{h}} \leq 0$. The wavefunction (27) is a solution for $|x|>L$ of the BdG equations up to second order derivatives. One can verify that for zero confining potential the solution (27), (28) is equivalent to the energy spectrum and coherence factors given in Eqs. (5)-(7).

For $\epsilon>\Delta_{0}$, the wavevectors $k^{\mathrm{e}, \mathrm{h}}$ are real at $|x|>L$, and hence the wavefunctions (27) remain properly bounded as $|x| \rightarrow \infty$. This is the continuous spectrum. The density of states $\rho^{\mathrm{e}, \mathrm{h}}(\epsilon)$ of the electron and hole 
independent of the precise behavior of $\Delta(\mathbf{r})$ in the constriction. Since $\arccos \left(\epsilon / \Delta_{0}\right)>0$, there is a single bound state per mode, with energy independent of the mode index $n \leq N$. This $N$-fold degenerate bound state at energy $\Delta_{0} \cos (\delta \phi / 2)$ differs qualitatively from the Andreev levels [24] in an SNS junction with $L_{\mathrm{N}} \gg \xi_{0}$, which are sensitive to the mode index, to the length $L_{\mathrm{N}}$ of the junction, and also to the pair-potential profile at the SN interfaces. This sensitivity is at the origin of the geometry-dependent critical current of the SNS junction which we discussed in the Introduction.

We are now ready to calculate the Josephson current from Eq. (20). (The alternative calculation based on Eq. (10) was given in Ref. [5], and leads to the same final result.) The integral over the continuous part of the excitation spectrum does not contribute in the limit $L / \xi_{0} \rightarrow 0$ (see above). The spatial integral of $|\Delta|^{2} / g$ contributes only over the region within the constriction $\left(|\Delta|\right.$ being independent of $\delta \phi$ in the reservoirs). Since $|\Delta|^{2} / g \sim$ $\Delta_{0} / \lambda_{\mathbf{F}} \xi_{0}$, one can estimate

$$
\frac{2 e}{\hbar} \int d \mathbf{r}|\Delta|^{2} / g \sim N\left(e \Delta_{0} / \hbar\right) \frac{L}{\xi_{0}}
$$

which vanishes in the limit $L / \xi_{0} \rightarrow 0$. What remains is the sum over the discrete spectrum in Eq. (20). Substituting $\epsilon_{p}=\Delta_{0} \cos (\delta \phi / 2), p=1,2, \ldots N$, we obtain the final result for the Josephson current through a quantum point contact:

$$
I(\delta \phi)=N \frac{e}{\hbar} \Delta_{0}(T) \sin (\delta \phi / 2) \tanh \left(\frac{\Delta_{0}(T)}{2 k_{\mathrm{B}} T} \cos (\delta \phi / 2)\right) .
$$

Since $N$ is an integer, Eq. (37) tclls us that $I$ for a given value of $\delta \phi$ increases stepwise as a function of the width of the constriction. At $T=0$ we have $I(\delta \phi)=N\left(e \Delta_{0} / \hbar\right) \sin (\delta \phi / 2)$, with a critical current $I_{\mathrm{c}}=$ $N e \Delta_{0} / \hbar$ (reached at $\delta \phi=\pi$ ). Near the critical temperature $T_{\mathrm{c}}$ we have $I(\delta \phi)=N\left(e \Delta_{0}^{2} / 4 \hbar k_{\mathrm{B}} T_{\mathrm{c}}\right) \sin (\delta \phi)$, and the critical current is reached at $\delta \phi=$ $\pi / 2$. The ratio $I / G$ (with $G=2 N e^{2} / h$ the normal-state conductance of the quantum point contact) does not contain $N$ and is formally identical to the result for a classical point contact $[\delta] .{ }^{5}$ In Fig. 3 we have plotted $I(\delta \phi)$ from $\mathrm{Eq}$. (37) for three different temperatures: $T=0,0.1,0.2$ in units of $\Delta_{0}(0) / k_{\mathrm{B}}=1.76 T_{\mathrm{c}}$. For these relatively low temperatures the $T$-dependence of $\Delta_{0}$ may be neglected. The $T$-dependence of the Josephson current is easily understood within the semiconductor model (cf. Sec. II), as a cancellation of the current due to the states in the energy gap at $-\epsilon_{p}$ and at $+\epsilon_{p}$. States above the Fermi energy are unoccupied at $T=0$, but become occupied when

\footnotetext{
${ }^{5}$ The identity of the classical and quantum iesults for $I / G$ should not be mistaken for a universal truth: It only holds within the approximations made in our analysis, and breaks down in particular in the transition region between two subsequent plateaux of discretized supercurrent.
} 
branches of the excitation spectrum is

$$
\begin{aligned}
\rho^{\mathrm{e}, \mathrm{h}} & =\frac{1}{2 \pi} \frac{d}{d \epsilon}\left(\int_{-L_{\mathrm{S}}}^{L_{\mathrm{S}}} k^{\mathrm{e}, \mathrm{h}} d x+\int_{-L}^{L} \delta k^{\mathrm{e}, \mathrm{h}} d x\right) \\
\delta k^{\mathrm{e}, \mathrm{h}} & =\left(2 m / \hbar^{2}\right)^{1 / 2}\left[U+\sigma^{\mathrm{e}, \mathrm{h}}\left(\epsilon^{2}-|\Delta|^{2}\right)^{1 / 2}\right]^{1 / 2}-k^{\mathrm{e}, \mathrm{h}} .
\end{aligned}
$$

The length $L_{S}$ of the superconducting regions at opposite sides of the constriction is assumed to be much larger than $\xi_{0}$. The wavevector change $\delta k^{\mathrm{e}, \mathrm{h}}$ is of order $1 / \xi_{0}$ for $\epsilon \gtrsim \Delta_{0}$ and decays as $\epsilon^{-3 / 2}$ for $\epsilon \gg \Delta_{0}$. It follows that the integral over the continuous spectrum in the expression (20) for the Josephson current is of order $N\left(e \Delta_{0} / \hbar\right) L / \xi_{0}$, which vanishes in the limit $L / \xi_{0} \rightarrow 0$.

For $0<\epsilon<\Delta_{0}$, the wavevectors $k^{\text {e, h }}$ have a non-zero imaginary part at $|x|>L$, so that Eq. (27) is not an admissible wavefunction for both $x>L$ and $x<-L$. The two bounded WKB wavefunctions for $0<\epsilon<\Delta_{0}$ are given at $|x|>L$ by

$$
\begin{aligned}
& \Psi_{+}= \begin{cases}A_{+} \Psi_{+}^{\mathrm{h}} & \text { if } x<-L, \\
B_{+} \Psi_{+}^{\mathrm{e}} & \text { if } x>L,\end{cases} \\
& \Psi_{-}= \begin{cases}A_{-} \Psi_{-}^{\mathrm{e}} & \text { if } x<-L, \\
B_{-} \Psi_{-}^{\mathrm{h}} & \text { if } x>L .\end{cases}
\end{aligned}
$$

The transition from $k^{\mathrm{h}}$ to $k^{\mathrm{e}}$ on passing through the constriction (associated with a change in sign of $\operatorname{Im~} k$ ) is analogous to Andreev reflection at an SN interface [24]. Andreev reflections are to be distinguished from ordinary reflections involving a change in sign of Re $k$. Ordinary reflections due to the pair potential are neglected in the WKB approximation. By matching the wavefunctions $\Psi_{ \pm}$to the region $|x|<L$ we obtain a boundary-value problem with a discrete energy spectrum. Since $\epsilon<\Delta_{0} \ll U$, we may approximate $k \approx \pm\left(2 m U / \hbar^{2}\right)^{1 / 2}$ in $\mathrm{Eq}$. (24) (the upper sign refers to $\Psi_{+}$, the lower sign to $\Psi_{-}$). The boundary-value problem then becomes

$$
\begin{aligned}
& \pm\left[\hbar^{2} U(x) / 2 m\right]^{1 / 2} \eta^{\prime}(x)+|\Delta(x)| \cos [\eta(x)-\phi(x)]=\epsilon, \\
& \eta(-L)=\phi_{1} \mp \arccos \left(\epsilon / \Delta_{0}\right), \\
& \eta(+L)=\phi_{2} \pm \arccos \left(\epsilon / \Delta_{0}\right) .
\end{aligned}
$$

Noting that $\eta$ is real, one deduces from Eq. (33) the inequality $\left|\eta^{\prime}\right|<$ $(\epsilon+|\Delta|)\left(\hbar^{2} U / 2 m\right)^{-1 / 2}$. Since $|\Delta| \lesssim \Delta_{0}$ and $U \gtrsim E_{\mathrm{F}}-E_{N}(0)$, we have the limiting behavior $|\eta(L)-\eta(-L)| \lesssim\left(L / \xi_{0}\right)\left(1-E_{N}(0) / E_{\mathrm{F}}\right)^{-1 / 2} \rightarrow 0$ in the limit $L / \xi_{0} \rightarrow 0$. Hence, to order $L / \xi_{0}$ the bound-state energy $\epsilon$ is determined by

$$
\arccos \left(c / \Delta_{0}\right)= \pm \frac{1}{2} \delta \phi
$$


independent of the precise behavior of $\Delta(\mathbf{r})$ in the constraction. Since $\arccos \left(\epsilon / \Delta_{0}\right)>0$, there is a single bound state per mode, with energy independent of the mode index $n \leq N$. This $N$-fold degenerate bound state at energy $\Delta_{0} \cos (\delta \phi / 2)$ differs qualitatively from the Andreev levels [24] in an SNS junction with $L_{N} \gg \xi_{0}$, which are sensitive to the mode index, to the length $L_{N}$ of the junction, and also to the pair-potential profile at the SN interfaces. This sensitivity is at the origin of the geometry-dependent critical current of the SNS junction which we discussed in the Introduction.

We are now ready to calculate the Josephson current from Eq. (20). (The alternative calculation based on Eq. (10) was given in Ref. [5], and leads to the same final result.) The integral over the continuous part of the excitation spectrum does not contribute in the limit $L / \xi_{0} \rightarrow 0$ (see above). The spatial integral of $|\Delta|^{2} / g$ contributes only over the region within the constriction ( $|\Delta|$ being independent of $\delta \phi$ in the reservoirs). Since $|\Delta|^{2} / g \sim$ $\Delta_{0} / \lambda_{\mathrm{F}} \xi_{0}$, one can estimate

$$
\frac{2 e}{\hbar} \int d \mathbf{r}|\Delta|^{2} / g \sim N\left(e \Delta_{0} / \hbar\right) \frac{L}{\xi_{0}},
$$

which vanishes in the limit $L / \xi_{0} \rightarrow 0$. What remains is the sum over the discrete spectrum in Eq. (20). Substituting $\epsilon_{p}=\Delta_{0} \cos (\delta \phi / 2), p=1,2, \ldots N$, we obtain the final result for the Josephson current through a quantum point contact:

$$
I(\delta \phi)=N \frac{e}{\hbar} \Delta_{0}(T) \sin (\delta \phi / 2) \tanh \left(\frac{\Delta_{0}(T)}{2 k_{\mathrm{B}} T} \cos (\delta \phi / 2)\right) .
$$

Since $N$ is an integer, Eq. (37) tells us that $I$ for a given value of $\delta \phi$ increases stepwise as a function of the width of the constriction. At $T=0$ we have $I(\delta \phi)=N\left(e \Delta_{0} / \hbar\right) \sin (\delta \phi / 2)$, with a critical current $I_{\mathrm{c}}=$ $N e \Delta_{0} / \hbar$ (reached at $\delta \phi=\pi$ ). Near the critical temperature $T_{\mathrm{c}}$ we have $I(\delta \phi)=N\left(e \Delta_{0}^{2} / 4 \hbar k_{\mathrm{B}} T_{c}\right) \sin (\delta \phi)$, and the critical current is reached at $\delta \phi=$ $\pi / 2$. The ratio $I / G$ (with $G=2 N e^{2} / h$ the normal-state conductance of the quantum point contact) does not contain $N$ and is formally identical to the result for a classical point contact [8]. ${ }^{5}$ In Fig. 3 we have plotted $I(\delta \phi)$ from Eq. (37) for three different temperatures: $T=0,0.1,0.2$ in units of $\Delta_{0}(0) / k_{\mathrm{B}}=1.76 T_{\mathrm{c}}$. For these relatively low temperatures the $T$-dependence of $\Delta_{0}$ may be neglected. The $T$-dependence of the Josephson current is easily understood within the semiconductor model (cf. Sec. II), as a cancellation of the current due to the states in the energy gap at $-\epsilon_{p}$ and at $+\epsilon_{p}$. States above the Fermi energy are unoccupied at $T=0$, but become occupied when

\footnotetext{
${ }^{5}$ The identity of the classical and quantum results for $I / G$ should not be mistaken for a universal truth: It only holds within the approximations made in our analysis, and breaks down in particular in the tiansition region between two subsequent plateaux of discretized supercurrent.
} 


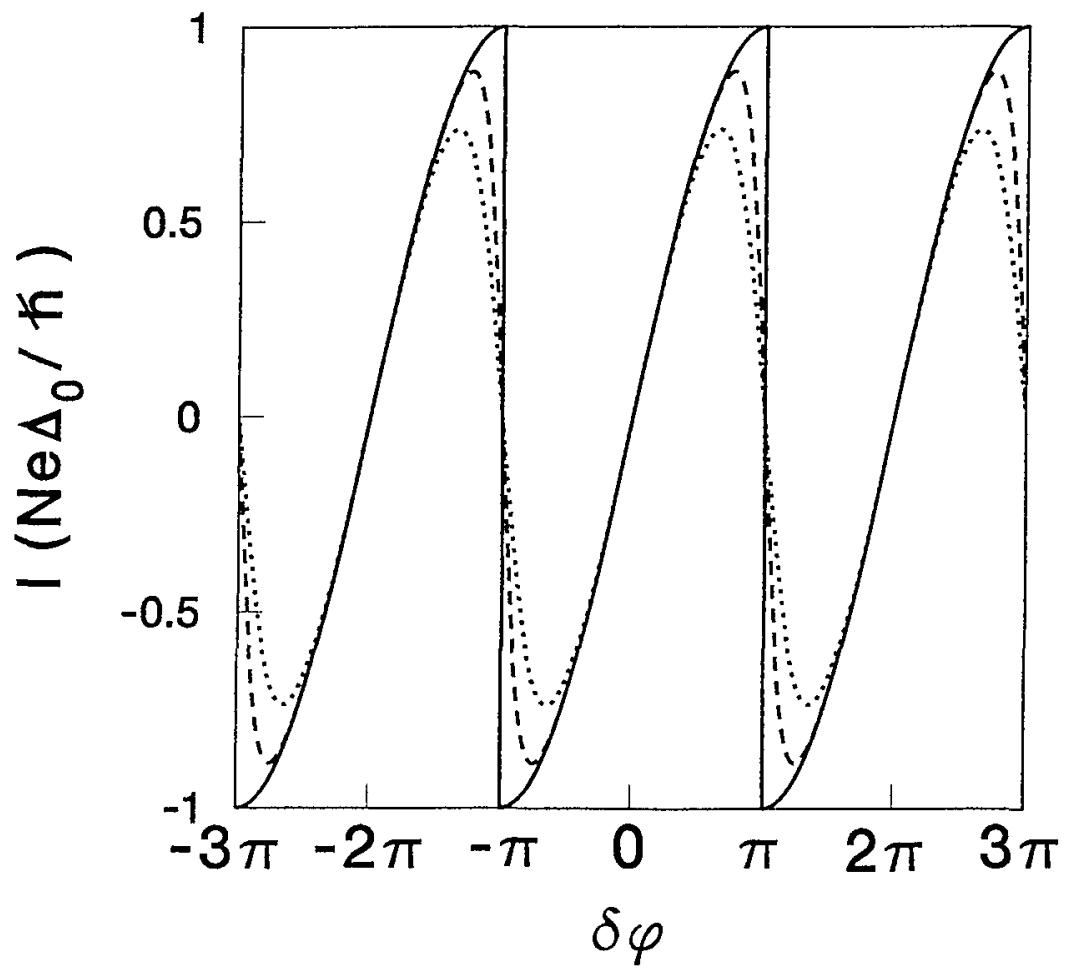

Figure 3: Current-phase relationship of a superconducting quantum point contact, according to $\mathrm{Eq}$. (37), for three different temperatures (in units of $\Delta_{0}(0) / k_{\mathrm{B}}$ ): $T=0$ (solid curve), $T=0.1$ (dashed curve), and $T=0.2$ (dotted curve).

$4 k_{\mathrm{B}} T \gtrsim \epsilon_{p}$. The supercurrent decays most rapidly near $|\delta \phi| \simeq \pi$, since $\epsilon_{p}$ is smallest there.

\section{EXPERIMENTAL REALIZATION}

A superconducting quantum point contact (SQPC) of fixed width might be constructed relatively easily as a constriction or microbridge in a superconductor of uniform composition. A test of the present theory, however, requires the fabrication of nanostructures with variable width or density. In the case of normal-state quantum point contacts this has been realized by depleting the high-mobility two-dimensional electron gas (2DEG) at the interface of a GaAs-AlGaAs heterostructure by applying a negative voltage to a split gate on top of the heterostructure $[1,2,25]$. This is an ideal system 


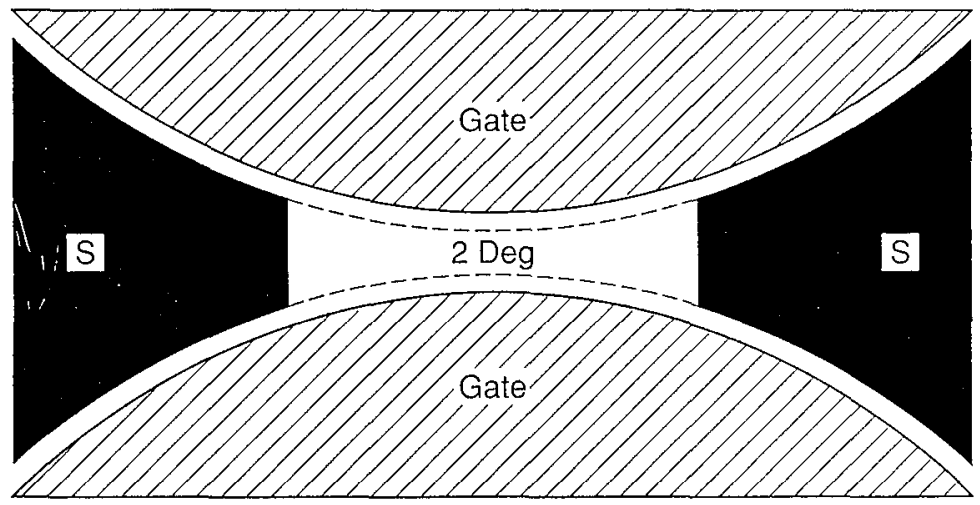

Figure 4: Top view of a possible experimental realization of a superconducting quantum point contact.

for the study of ballistic transport of normal electrons, because of the long mean free path $(l \simeq 10 \mu \mathrm{m})$ and Fermi wave length $\left(\lambda_{\mathrm{F}} \simeq 50 \mathrm{~mm}\right)$.

An SQPC might be realized in the same system, if superconducting contacts to the 2DEG can be made [26]. An observation of the discretized critical current requires that the superconducting regions extend well into the constriction on either side, as illustrated schematically in Fig. 4 (black regions). A remaining problem is the mismatch in Fermi energy that will in general exist at the interface between the 2DEG (where $E_{\mathrm{F}} \simeq 10 \mathrm{meV}$ ) and the superconductors (where $E_{\mathrm{F}} \simeq 1 \mathrm{eV}$ ). This mismatch, if it is abrupt, induces normal reflections (rather than Andreev reflections) of quasiparticles incident on the reservoirs. The fabrication of sufficiently clean superconducting contacts to the 2DEG in a GaAs-AlGaAs heterostructure may be difficult, because contacting requires an alloying process. This problem may be circumvented by using the surface 2DEG present as a natural inversion layer on p-InAs [27]. Superconducting contacts, with a shape as in Fig. 4, may then be evaporated directly onto the surface. Once the contacts have been made, a constriction of variable width may again be realized by means of split gates (insulated from the surface 2DEG). Because of the non-linear dependence of the supercurrent on the gate voltage, such an SQPC has possible device applications [28].

\section{References}

[1] B. J. van Wees, H. van Houten, C. W. J. Beenakker, J. G. Williamson, L. P. Kouwenhoven, D. van der Marel, and C. T. Foxon, Phys. Rev. Lett. 60, 848 (1988). 
[2] D. A. Wharam, T. J. Thornton, R. Newbury, M. Pepper, H. Ahmed, J. E. F. Frost, D. G. Hasko, D. C. Peacock, D. A. Ritchie, and G. A. C. Jones, J. Phys. C 21, L209 (1988).

[3] H. van Houten and C. W. J. Beenakker, in: Analogıes ın Optıcs and Micro-Electronıcs, edited by W. van Haeringen and D. Lenstra (Kluwer, Dordrecht, 1990).

[4] E. A. Montie, E. C. Cosman, G. W. 't Hooft, M. B. van der Mark, and C. W. J. Beenakker, Nature 350, 594 (1991).

[5] C. W. J. Beenakker and H. van Houten, Phys. Rev. Lett. 66, 3056 (1991).

[6] H. van Houten and C. W. J. Beenakker, in: Proc. Int. Symp. on Analogies in Optıcs and Micro-Electronucs, edited by D. Lenstra and W. van Haeringen (North-Holland, Amsterdam, to be published); Physica B\&C (to be published).

[7] For a review of the Josephson effect in weak links, see: K. K. Likharev, Rev. Mod. Phys. 51, 101 (1979).

[8] I. O. Kulik and A. N. Omel'yanchuk, Fiz. Nisk. Temp. 3, 945 (1977); 4, 296 (1978) [Sov. J. Low Temp. Phys. 3, 459 (1977); 4, 142 (1978)].

[9] Yu. V. Sharvin, Zh. Eksp. Teor. Fiz. 48, 984 (1965) [Sov. Phys. JETP 21, 655 (1965)].

[10] B. D. Josephson, Phys. Lett. 1, 251 (1962).

[11] P. W. Anderson, in: Ravello Lectures on the Many-Body Problem, edited by E. R. Gianello (Academic, New York, 1963).

[12] V. Ambegaokar and A. Baratoff, Phys. Rev. Lett. 10, 486 (1963); 11, 104(E) (1963).

[13] L. G. Aslamazov, A. I. Larkin, and Yu. N. Ovchinnikov, Zh. Eksp. Teor. Fiz. 55, 323 (1968) [Sov. Phys. JETP 28, 171 (1969)].

[14] I. O. Kulik, Zh. Eksp. Teor. Fiz. 57, 1745 (1969) [Sov. Phys. JETP 30, $944(1970)]$.

[15] C. Ishii, Prog. Theor. Phys. 44, 1525 (1970).

[16] A. V. Svidzinsky, T. N. Antsygina, and E.N. Bratus', Zh. Eksp. Teor. Fiz. 61, 1612 (1971) [Sov. Phys. JETP 34, 860 (1972)]; J. Low Temp. Phys. 10, 131 (1973). 
[17] J. Bardeen and J. L. Johnson, Phys. Rev. B 5, 72 (1972).

[18] A. Furusaki, H. Takayanagi, and M. Tsukada (preprint).

[19] L. I. Glazman, G. B. Lesovik, D. E. Khmel'nitskii, and R. I. Shekhter, Pis'ma Zh. Eksp. Teor. Fiz. 48, 218 (1988) [JETP Lett. 48, 238 (1988)]

[20] A. Yacoby and Y. Imry, Phys. Rev. B 41, 5341 (1990).

[21] P. G. de Gennes, Superconductivity of Metals and Alloys (Benjamin, New York, 1966).

[22] M. Tinkham, Introductıon to Superconductıvity (McGraw-IIill, New York, 1975).

[23] J. Bardeen, R. Küümel, A. E. Jacobs, and L. Tewordt, Phys. Rev. 187, $556(1969)$.

[24] A. F. Andreev, Zh. Eksp. Teor. Fiz. 46, 1823 (1964); 49, 655 (1965) [Sov. Phys. JETP 19, 1228 (1964); 22, 455 (1966)].

[25] For a review of the field of quantum transport in semiconductor nanostructures, see: C. W. J. Beenakker and H. van Ilouten, Solid State Physics 44, 1 (1991).

[26] Z. Ivanov, T. Claeson, and T. Andersson, Jpn. J. Appl. Phys. Suppl. 26-3, 1617 (1987).

[27] H. Takayanagi and T. Kawakami, Phys. Rev. Lett. 54, 2449 (1985).

[28] H. van Ilouten, Appl. Phys. Lett. 58, 1326 (1991). 